\title{
Multicenter Study of Staging and Therapeutic Predictors of Hepatocellular Carcinoma Recurrence following Transplantation
}

Theodore H. Welling ${ }^{1}$, Kevin Eddinger ${ }^{2}$, Kristen Carrier ${ }^{3}$, Danting Zhu ${ }^{4}$, Tyler Kleaveland ${ }^{5}$, Derek E. Moore ${ }^{6}$, Douglas E. Schaubel ${ }^{4}$, and Peter L. Abt ${ }^{2}$

Perlmutter Cancer

er Center, NYU Langone Health, NY, NY; ${ }^{2}$ Department of Surgery, Hospital of the University of Pennsylvania; Schools of ${ }^{3}$ Pharmacy, ${ }^{4}$ Public Health, and ${ }^{5}$ Medicine, University of Michigan, Ann Arbor, MI; ${ }^{6}$ St.Thomas Health, Nashville, TN

Keywords: liver cancer, transarterial chemoembolization, local-regional treatment, down-staging, pathologic response

Theodore H. Welling Theodore.Welling@nyumc.org

Kevin Eddinger eddinger@mail.med.upenn.edu

Kristen Carrier klcarr@med.umich.edu

Danting Zhu dantzhu@umich.edu

Tyler Kleaveland kleavelt@umich.edu

Derek E. Moore Derek.Moore@ascension.org

Douglas E.Schaubel deschau@umich.edu

Peter L.Abt Peter.Abt@uphs.upenn.edu

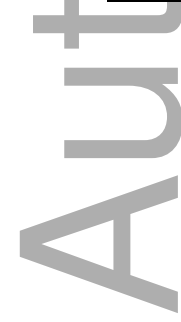

This is the author manuscript accepted for publication and has undergone full peer review but has not been through the copyediting, typesetting, pagination and proofreading process, which may lead to differences between this version and the Version of Record. Please cite this article as doi: $\underline{10.1002 / \text { lt.25194 }}$

This article is protected by copyright. All rights reserved 


\section{Footnotes}

Contact Information:

Theodore $\vec{H}$. Welling, MD

Associate Professor of Surgery

Director, Liver Tumor Program

Perlmutter Cancer Center

NYU Langone Health

Alexandria Center for Life Science West

430 E. $29^{\text {th }}, 660 \mathrm{~B}$

New York, NY 10016

(646) 754-2672, ext 42672

(646) 501-4564 (fax)

Theodore.Welling@nyumc.org

Abbreviations: OLT, orthotopic liver transplantation; HCC, hepatocellular carcinoma; MELD, model for end-stage liver disease; TACE, transarterial chemoembolization; HR, hazard ratio; RFA, radiofrequency ablation; $\mathrm{cm}$, centimeter; HCV, hepatitis C; NASH, non-alcoholic steatohepatitis; UNOS, United Network for Organ Sharing; SRTR, Scientific Registry of Transplant Recipients; IRB, institutional review board; mRECIST, modified response evaluation criteria in solid tumors; AASLD, American Association for the Study of Liver Diseases; AFP, alpha-fetoprotein;

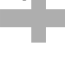

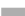

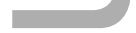

Financial Support: Biocompatibles Inc., a BTG International group company. 


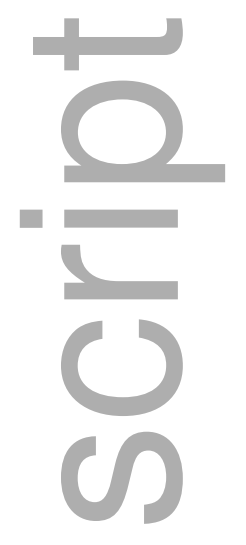

Abstract

Orthotopic liver transplantation (OLT) and resection are effective treatments for hepatocellular carcinoma (HCC). However, optimizing OLT and limiting $\mathrm{HCC}$ recurrence remains a vexing problem. New HCC MELD and allocation algorithms provide greater observation of HCC patients, many while receiving local-regional treatments. Potential benefits of local-regional treatment for limiting HCC recurrence post-OLT remain incompletely understood. Therefore we aimed to define $\mathrm{HCC}$ specific prognostic factors affecting recurrence in a contemporary, multicenter cohort of $\mathrm{HCC}$ patients undergoing OLT and specifically whether local-regional therapies limited recurrence. We identified 441 patients undergoing OLT for HCC at three major transplant centers from 2008-2013. Cox regression was used to analyze covariate-adjusted recurrence and mortality rates post-OLT. "Bridging" or "down-staging" therapy was used in 238 patients $(54 \%)$ with transarterial chemoembolization (TACE) being used in $170(71 \%)$ of treated patients. The survival rate post-OLT was $88 \%$ and $78 \%$ at 1 and 3 years, respectively, with $\mathrm{HCC}$ recurrence $(28 \%$ of deaths) significantly increasing mortality rate $(H R=19.87, p<0.0001)$. Tumor size, not tumor number, either at presentation or on explant independently predicted HCC recurrence (HR 1.36 and 1.73, respectively, $p<0.05$ ) with a threshold effect noted at 4.0 $\mathrm{cm}$ size. Local-regional therapy (TACE) reduced HCC recurrence by $64 \%$ when adjusting for presenting tumor size $(\mathrm{HR} 0.36, \mathrm{p}<0.05)$. Explant tumor size and microvascular invasion predicted mortality (HR 1.19 and 1.51 , respectively, $\mathrm{p}<0.05)$ and pathologic response to therapy (TACE or RFA) significantly decreased explant tumor size $(0.56-1.62 \mathrm{~cm}$ diameter reduction, $\mathrm{p}<0.05)$. Conclusion: $\mathrm{HCC}$ tumor size at presentation or explant is the most important predictor 
for HCC recurrence post-OLT. Local-regional therapy to achieve a pathologic response (decreasing tumor size) can limit HCC recurrences post-OLT.

\section{(r)}

\section{Introduction}

Hepatocellular carcinoma (HCC) continues to be a significant cause of mortality among all other solid malignancies and is noted to have the fastest rising incidence and mortality in the U.S. among other cancers (1). HCC is most commonly associated with chronic liver disease or cirrhosis, with Hepatitis $\mathrm{C}$ virus (HCV) and increasingly non-alcoholic steatohepatitis (NASH) being common underlying etiologies (2-4). Therefore, orthotopic liver transplantation (OLT) has been utilized as a means to effectively treat HCC and underlying liver disease. However, mortality related to HCC recurrence following OLT has continued to remain significant. New HCC allocation policies have recently been implemented to allow for observation time and implementation of local regional therapies for HCC (5). Outcomes supporting the rationale for this policy have shown that a greater duration of time following HCC presentation to OLT is protective against mortality following OLT for HCC patients (6-8).

While these policies predicated on wait time may improve overall survival for HCC patients undergoing OLT, it is still unclear which HCC related factors are most likely to influence HCC specific outcomes such as HCC recurrence. Specifically, the influence of factors related to initial HCC presentation, final pathology, and benefits of local-regional therapy such as radiofrequency ablation (RFA) or transarterial chemoembolization (TACE) are incompletely defined. Prior studies are either conflicting, lack specific evaluation of these important variables, or are under powered to fully evaluate their impact on HCC recurrence following OLT (9-12). Commonly utilized databases (e.g., United Network for Organ Sharing (UNOS); Scientific Registry of Transplant Recipients (SRTR)) do not provide detailed data with respect to imaging (pre- and post- therapy), explant pathology with respect to response, or specific follow up with respect to HCC disease recurrence (13-15). Others studies represent those of single centers and while informative, may not be translatable to other centers or lack untreated controls to specifically evaluate the role of local-regional treatments (16). 
Both RFA and TACE are known to provide a survival benefit in appropriately selected HCC patients based upon HCC tumor stage (17-20). Furthermore, recent technical advances in how TACE is implemented (superselective/selective versus lobar) appear to have improved HCC tumor responses in more contemporary studies (21). However the specific effects of these therapies in patients who ultimately undergo OLT for $\mathrm{HCC}$ on $\mathrm{HCC}$ recurrence remain controversial.

口

We therefore aimed to better understand and inform future liver allocation policy decisions and treatment strategies for HCC patients who are potential OLT candidates. Thus, we sought to more completely identify the effects of disease presentation, treatments, and response to treatments on HCC recurrence following OLT. A multicenter, retrospective cohort of HCC patients who underwent OLT at three major transplant centers was examined. We identified which $\mathrm{HCC}$ disease factors at initial presentation and the success of local regional therapies predicted HCC recurrence following OLT in this multicenter cohort.

\section{Experimental Procedures}

\section{Patient Data Extraction}

The study was conducted after IRB approval at all study sites. No donor organs were obtained from executed prisoners or other institutionalized persons. Consecutive patients from 20082013 undergoing OLT from three major contemporary transplant centers (University of Pennsylvania, University of Michigan, Vanderbilt University) were identified with a diagnosis of HCC confirmed on explant pathology. HCC and liver disease characteristics were determined at the time of initial diagnosis of HCC (not formal evaluation of OLT) and at the time of OLT. Patients were required to have at least two years of follow up following OLT. Other diagnoses discovered on explant pathology (e.g. cholangiocarcinoma or mixed HCC-cholangiocarcinoma) were not included in the cohort. Patients who underwent local regional treatment were excluded from the cohort if there was incomplete treatment details, such as those treated at outside centers before presentation. Patients were not included if their follow up was at a center different from that at which they received the OLT. Patients were listed for OLT at the three centers if they met Milan criteria. 
Patient data included age, sex, liver disease etiology, laboratory Model for End-stage Liver Disease (MELD) score, MELD exception status at OLT, listing date, and date of OLT were recorded. HCC presenting tumor burden or stage was determined by examining CT or MRI reports and utilizing mRECIST criteria for diagnosis of HCC according to American Association for the Study of Liver Diseases (AASLD) guidelines. Subsequent radiographic reports were similarly characterized either following local-regional therapies or at the time closest to OLT, depending on time elapsed between initial evaluation or treatment and OLT. Serum alphafetoprotein (AFP) was also serially determined at presentation and at time of OLT. Explant tumor pathology regarding stage was determined by review of pathology reports and recording of maximal tumor size, tumor number, and presence or absence of microvascular invasion. Only viable tumor tissue on explant was used to characterize tumor number and size on final pathology and thus pathologic stage. For example, tumors which were completely necrotic (100\% necrotic), this was equated to a pathologic complete response for which the viable tumor diameter would be zero centimeters. Maximal radiographic and explant pathologic response was recorded if local-regional therapy was used. Responses were characterized as either no response, partial response, or complete response.

\section{(a)}

Characteristics regarding presence or absence of local regional therapies were also recorded, as were type of therapy used (e.g. TACE, RFA, radiation), individual characteristics of TACE (such as selective versus non-selective and type of agent used). Decisions regarding use of local regional therapies were made at the discretion of individual centers and incorporated "down-staging" or "bridging" intent as recommended by guidelines using BCLC criteria (18).

\section{Statistical Analysis}

For descriptive analysis of the study cohort, numeric variables were summarized by the sample mean, while percentages were used for categorical covariates. Cox regression was used to analyze covariate-adjusted recurrence rates. Death was treated as a competing risk, in the sense that the hazard rate for recurrence applied to patients actually at risk for recurrence (i.e., alive, with no previous recurrence), known as the cause-specific hazard in the competing risks literature (22). 
Five different HCC recurrence rate models were fitted. These were designed to specifically isolate whether particular co-variates might influence HCC recurrence. All five models adjusted for age, sex, etiology, AFP at OLT, lab MELD at OLT, difference between HCC exception MELD score and lab MELD score, and time between initial HCC presentation and OLT. The individual models were characterized by which of the remaining co-variates were included, which depended on the particular goal or hypothesis each model was designed to ascertain for an effect of a co-variate on HCC recurrence. Specifically, Model 1 focused on initial presentation of tumor characteristics (tumor number, size of largest tumor) based on radiography. Model 2 instead focused on the same tumor characteristics, but based on information available on explant pathology; the model also included an indicator for microvascular invasion (1=yes; $0=$ no) and calendar year of diagnosis. Model 3 evaluated initial tumor characteristics (size, number), and also the rate of change of such characteristics, as well as calendar year. Model 4 sought to describe the effect of calendar year as a means of describing recurrence trends over time. Due to the lack of adjustment for tumor characteristics or treatment type, the calendar year of diagnosis effect estimated through Model 4 is expected to represent the aggregate effects of potential changing trends in HCC diagnosis and treatment over time. Finally, Model 5 contained co-variates for initial tumor size and number, as well as local regional treatment category (RFA, TACE, RFA and TACE, or no treatment).

Additional, separate proportional hazards models (Models 1-3, Table 3) were also fitted to the outcome of death. Note that death was not considered censored for patients who experienced recurrence. Death was censored as a separate outcome as patients had deaths as an endpoint due to HCC recurrence but also due to non-HCC related causes as well. Similar to HCC recurrence as an outcome, a set of mortality outcome models was fitted with the same covariates for Models 1-3 in Table 3. Separate models for calendar year of diagnosis or treatments with mortality as the outcome were not created for mortality analysis.

We then fitted an additional separate Cox model for death which included a time-dependent binary $(0,1)$ covariate for HCC recurrence. At OLT, all patients had the recurrence covariate set to 0 ; for patients who experienced recurrence, the covariate switched to 1 at the time of recurrence and remained at 1 thereafter. The purpose of this model was to quantify the covariate-adjusted effect of HCC recurrence on subsequent mortality. 
Additionally we designed linear regression models (Models 1-2, Table 4) to specifically analyze the effect of covariates to determine final tumor size on explant pathology. Co-variates were those noted at the time of OLT or initial presentation (Model 1). Model 2 includes the same covariates but also types of local-regional treatments (versus no treatment) to the linear regression analysis.

\section{Results}

Baseline Clinical Characteristics

A total of 441 transplant patients were identified with a diagnosis of HCC during the interval of 2008 to 2013 that met the defined inclusion and exclusion criteria. Their baseline clinical characteristics are presented in Table 1. As expected, the majority of patients were male by a nearly $4: 1$ ratio and hepatitis $C$ was the most common underlying liver disease etiology (69.5\%) with NASH/cryptogenic and alcohol making up the next most common etiologies, $10.4 \%$ and $8.8 \%$, respectively. The median follow up for the entire cohort was 47.3 months following transplant. Median AFP at initial presentation was $15.5 \mathrm{ng} / \mathrm{mL}$. Patients who initially presented outside of Milan criteria were $11.4 \%$ of the entire cohort. The mean number of tumors at presentation was 1.4 and the mean size at presentation was $2.6 \mathrm{~cm}$. "Bridging" or "downstaging" local regional TACE or RFA therapy for HCC was used in 238 (54\%) of HCC patients and TACE was the most common local-regional therapy used, comprising $170(71 \%)$ of the patients treated. RFA was the next most common modality, comprising 51 (21\%) of treated patients, and combined TACE and RFA comprised 17 (7\%) of treated patients. Other treatments were used rarely in this cohort and included XRT (2 patients), Y90 (1 patient), resection (1 patient), and ethanol ablation ( 2 patients). Fifteen patients not treated with local regional therapy had incidentally discovered HCC on explant of which eleven (73.3\%) were within Milan criteria. The mean time following initial HCC presentation to OLT was 11.4 months. The survival rate following OLT was $88 \%$ and $78 \%$ at 1 and 3 years post- OLT, respectively (Figure 1). There was an overall thirty percent mortality rate for the cohort due to all causes (133 patients) of which HCC recurrence accounted for 37 of these deaths. Thus, HCC recurrence was the cause for mortality in $28 \%$ of patient deaths post-transplant.

\section{Predictors of HCC Recurrence Following Transplant}

Results of the Cox regression models for HCC recurrence rate are in Table 2. Initial modeling (Table 2, Model 1) showed that baseline etiology of liver disease, age, and sex had no apparent 
effect on HCC recurrence following OLT. Incidentally discovered HCC patients $(n=15)$ had an HCC recurrence rate of $13.3 \%$ ( $n=2$ patients). The difference between lab MELD score and MELD HCC exception score at OLT was a significant predictor of HCC recurrence, such that there was $7 \%$ decrease in recurrence rate for every lab MELD unit decrease in score. Maximum initial tumor size also predicted recurrence, with a calculated $36 \%$ increase in the $\mathrm{HCC}$ recurrence rate for every $1.0 \mathrm{~cm}$ increase in tumor size at presentation. The number of HCC tumors at presentation did not significantly predict recurrence. When explant pathology characteristics were substituted into the model for initial tumor presentation (Table 2, Model 2), maximum tumor size had an even greater effect on the recurrence rate, with a $73 \%$ increase for every $1.0 \mathrm{~cm}$ increase in tumor size. The number of tumors found on explant pathology likewise had no association with recurrence. Microvascular invasion identified on explant pathology had a significant, 4.54 fold increased risk for HCC recurrence rate following OLT, similar to previous studies. When maximum tumor sizes were taken into account both at initial presentation and at the time of OLT (Table 2, Model 3), evidence of progression (increasing tumor size) had the most profound effect such that there was a greater than six-fold increase in HCC recurrence rate for every $1.0 \mathrm{~cm}$ of maximum tumor size progression.

To further delineate the effect of explant pathology maximum tumor size on overall HCC recurrence rate, we grouped explant maximal tumor sizes into quintiles in order to nonparametrically assess the effect of tumor size on HCC recurrence rate (Figure 2). Indeed, the effect of maximum tumor size appeared to be non-linear with an apparent threshold effect at 4.0 $\mathrm{cm}$ tumor size to predict recurrence following OLT. Note that, based on radiographic images at initial presentation, the functional relationship between maximum tumor size and HCC recurrence rate was less clear and demonstrated no threshold effect (data not shown).

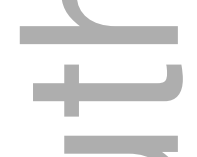

The year of HCC diagnosis at initial presentation appeared to effect overall HCC recurrence rate following OLT (Table 2, Model 4) when initial presenting tumor characteristics and explant findings were removed from the model. Therefore, HCC recurrence rate decreased by $19 \%$ for every yearly increment in calendar year of diagnosis. Thus improving aggregate trends in HCC diagnosis and treatment were observed over time for this cohort as this model was not adjusted for tumor characteristics and local-regional treatments. 
Factors that predicted a lower HCC recurrence rate, other than smaller tumor size on initial presentation or on final pathology and independent of wait time showed that local-regional treatment for $\mathrm{HCC}$ had a lower predicted $\mathrm{HCC}$ recurrence rate (Table 2, Model 5). Specifically, use of TACE reduced HCC recurrence by $64 \%$ when adjusting for initial tumor size at presentation. Thus when controlled for initial tumor size, the rate of $\mathrm{HCC}$ recurrences per 100 patient years was reduced from 2.26 recurrences down to 1.72 recurrences with local-regional treatment. Use of RFA did not appear to predict a lower recurrence rate except when accounting for radiographic response such that complete absence of a radiographic response predicted as much as a 15 -fold increase in recurrence rate. Of note, for patients receiving RFA, $11.5 \%$ had tumors over 3.5 centimeters and $17 \%$ had tumors over 3.0 centimeters in size. Combined use of RFA and TACE did not reach statistical significance toward reducing HCC recurrence rated following OLT. Other treatment modalities, such as radiation therapy or Y90, were used in too small of a sample size to permit analysis.

\section{Factors Associated with Mortality in HCC Patients Following OLT}

Since mortality following OLT can occur due to non-HCC related complications of OLT, we sought to identify whether any of the HCC related patient factors were predictive of overall mortality. This is important from the perspective that HCC recurrence accounted for the cause of death in $28 \%$ of all patient deaths post-OLT. In this multivariate analysis, with death as the endpoint, patient factors such as sex and etiology were not predictive for mortality (Table 3). However, more advanced age incrementally increased mortality by $16-20 \%$ for every five year increment (Table 3, Models 1-3). With respect to HCC related factors, tumor number at initial presentation or on pathology, not tumor size at initial presentation, predicted mortality with a 26$28 \%$ increase in death rate per tumor (Table 3, Model 1 and Model 3). However, based on information available at explant pathology (Table 3, Model 2), number of tumors was no longer associated with mortality, while maximum tumor size did exhibit a significant association (19\% increase in mortality rate per $\mathrm{cm}$ ). Microvascular invasion was associated with a $51 \%$ increase in mortality rate, a result which approached but did not attain statistical significance $(p=0.07)$. Elevated AFP, particularly greater than $1,000 \mathrm{ng} / \mathrm{mL}$, predicted a higher mortality rate following OLT consistent with prior reports showing an effect on HCC recurrence and survival (Table 3 , Model 1) $(23,24)$. Likewise, when AFP increased between initial presentation and time of OLT (Table 3, Model 2), this had a more profound association with mortality with over a three-fold 
effect. The model with $\mathrm{HCC}$ recurrence as a time-dependent predictor estimated that HCC recurrence increases mortality almost 20 -fold ( $H R=19.87, p<0.0001)$.

\section{Predictors of HCC Explant Pathology}

Since HCC explant pathology, particularly final tumor size, had the most profound effect on $\mathrm{HCC}$ recurrence rate and is the most potentially modifiable $\mathrm{HCC}$ related factor, we sought to isolate which factors might overall influence this effect utilizing explant pathology as the endpoint. In Table 4, two linear regression models were designed to evaluate factors affecting size of the largest tumor (cm diameter) at explant. Both models evaluate characteristics at the time of OLT. However Model 2 also includes HCC treatment categories. Based on Model 1, the significant predictors of tumor size at explant are initial presenting tumor size (positive sign=increasing tumor size) and calendar year of diagnosis (negative sign=decreasing tumor size). Model 2, which included treatments, showed increased initial tumor size still significantly increases explant size, while explant size still decreased significantly with increasing calendar year of diagnosis. In addition, Model 2 reveals that, relative to no HCC treatment, RFA treatment, TACE treatment, or combined RFA and TACE treatment were significantly associated with decreased maximal tumor size at explant. The effect of receiving both RFA and TACE was greater than the sum of RFA-alone and TACE-alone, indicating a potential synergistic effect on decreasing tumor size at explant.

Responses to treatment was classified as either none, partial, or complete. For the 238 patients treated with either RFA or TACE, $50 \%$ had a pathologic response at least as favorable as the radiographic response. The proportion was slightly higher for patients treated with TACE (at $52 \%$ ) compared to those receiving RFA (44\%).

\section{Discussion}

Recent studies have led to a change in HCC OLT allocation policy such that an observation period is beneficial before OLT once a radiographic diagnosis of HCC is established (5-8). This presumably offers time for 1 ) the individual HCC biology to declare itself, 2) the radiographic stage to be secured, 3) perhaps allow local-regional therapies to identify patients who may or may not benefit from OLT, and 4) control disease while other work up and listing proceeds for OLT. Indeed, local-regional therapies such as RFA and TACE are well established to create a 
pathologic and radiographic response in many patients, translating into a survival benefit when properly deployed $(17,18,20)$. However, the benefit of local-regional therapy during this observation period toward limiting HCC recurrences and thus HCC-related mortality is not completely defined, particularly in the context of other known prognostic predictors for HCC recurrence.

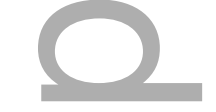

The present study identified that HCC tumor stage, particularly tumor size was one of the most important predictors for both HCC recurrence and mortality. HCC recurrence was overall the most significant predictor for mortality for all patients undergoing OLT for HCC. We have shown, similar to prior studies, that microvascular invasion was one of the most_significant predictors of HCC recurrence, independent of tumor size (24). AFP elevation, either at the time of transplant or the rate of AFP increase, had no independent effect on HCC recurrence when tumor size was included in the multivariate models. Later calendar year of diagnosis had an independent effect of lowering the risk of HCC recurrence. This is likely related to either improved diagnostics over time or the increasing use of local-regional therapies over time in this multicenter cohort. Indeed, inclusion of TACE treatment to the models showed a reduction of HCC recurrence when compared to no local-regional_treatment and also when initial presenting tumor size was included in the model. This effect of TACE was independent of time from initial presentation to the time of OLT (total wait time) (Table 2, Model 5). Indeed, time from presentation of HCC to the time of OLT was not independently predictive in the models for HCC recurrence (Table 2) whereas treatment, initial tumor size, tumor progression, and pathologic characteristics were independently predictive, suggesting that factors other than "wait time" were important for predicting HCC recurrence, particularly tumor stage and treatment.

Use of local-regional therapies increased over time, likely initially affected by lower transplant waiting times in the included eras for participating centers during the early part of this cohort. Thus the total proportion of patients receiving local-regional treatment was less than a recent UNOS data base study (25). However, the present study had a much longer median follow up of 47.3 months, takes into account initial HCC presentation stage, and subsequent management. Prior studies such as the UNOS study assess stage from the time of transplant listing filing which is often dependent on many other medical and social factors. The inclusion of a non-treated HCC group also serves as an additional control allowing the current study to isolate potential separate effects of local-regional treatment on HCC related outcomes. 
Concordant to previous studies, high AFP levels (greater than $1000 \mathrm{ng} / \mathrm{mL}$ ), along with rate of AFP increase, were independently associated with a higher mortality rate $(23,24,26)$. As to why lower levels do not correlate independently, this is likely due to AFP (in AFP producing HCCs) to correspond to tumor burdens (23) and not necessarily signifying a more aggressive $\mathrm{HCC}$. This has been shown in the resection literature with respect to tumor stage, not AFP, to independently predict worse prognoses (27). A similar relationship of microvascular invasion to tumor size has also been noted (23), but presence of microvascular invasion in the current study and others was a strong independent risk factor. Indeed, there was a threshold effect (Figure 2) for tumor size diameter of $4.0 \mathrm{~cm}$ to increase the risk of HCC recurrence. Thus tumor size, either radiographically or on final pathology, appears to be the best measurable and perhaps most modifiable factor toward limiting HCC recurrences with respect to the role of localregional therapies. This suggests that the greatest benefits for local regional therapy may be derived when tumors greater than or equal to $4.0 \mathrm{~cm}$ are treated such that at least a partial response may be generated to reduce tumors to less than $4.0 \mathrm{~cm}$ in size.

TACE showed a benefit with respect to limiting HCC recurrence on the Cox modeling analysis. RFA did not independently predict a decrease in HCC recurrence except in cases where there was a complete absence of a response in which these patients carried a 15-fold increase in recurrence rate. The lack of significance for RFA to independently decrease HCC recurrence is unclear as RFA has a known, potentially curative, therapeutic benefit when properly applied to tumors less than $3.5 \mathrm{~cm}$ in size $(19,20)$. Since this study is retrospective, possibilities are that either 1) RFA may have been applied to tumors at the borderline of this threshold in that $11.5 \%$ of RFA patients had tumors greater than $3.5 \mathrm{~cm}$ and $17 \%$ had tumors greater than $3.0 \mathrm{~cm}$, or 2) the number of patients receiving RFA alone may have been under powered to detect a statistical difference. Indeed, we did note twelve patients who had RFA alone and had a complete pathologic response and no HCC recurrences with four patients dying due to nonHCC related causes (data not shown). However, the number was small and failed to be statistically significant when accounting for other factors.

This article is protected by copyright. All rights reserved 
Tumor size on explant was the most significant, objectively measured, tumor related factor predicting mortality. Microvascular invasion approached, but did not quite achieve, statistical significance when adjusting for other factors and when tumor size at explant was maintained in the model. This could be due to the known correlation between increasing tumor burden (size) and vascular invasion $(23,27)$. Given the importance of explant pathology tumor size to predict both HCC recurrence and overall mortality, we used this endpoint to assess pathologic response of the local-regional therapies. In this analysis, RFA, TACE, or combination (TACE and RFA) versus no treatment had a benefit to reducing tumor burden. This was independent of both calendar year of diagnosis and initial tumor size, suggesting an important effect of localregional therapy alone. In at least $50 \%$ of patients where a pathologic response was noted, the radiographic response was at least as favorable, suggesting that radiographic response may serve as a useful correlate in future studies when trying to evaluate success of local-regional therapies and ultimately aiding in determining OLT liver allocation algorithms. However, these correlations would need to be validated in a prospectively designed study. These findings in our present study are in concordance with a recent single center study evaluating the importance of pathologic response (vs. lack of a response) to predict less HCC recurrence (16). However, the previously referenced study did not contain an untreated group to serve as a control whereas our present study had the advantage of containing an untreated group for comparison.

The present study is retrospective and does not allow for independent, prospective review of radiographic response and indeed in some patients was not able to be performed in time before patients underwent OLT shortly following local-regional therapy. Likewise, independent, prospective pathology review was not possible given the retrospective nature and thus assessments of presence or absence of microvascular invasion could be discordant (28). Tumor size, either on pathology or on radiographic evaluation, is accepted to be a relatively objective measurement in oncologic studies. Additionally, treatment bias may be present given the retrospective nature of this study cohort. However, the current study does represent contemporary practice among three high volume liver transplant centers and detailed data regarding tumor characteristics--particularly size, HCC recurrence, and HCC-specific factors were able to be reliably obtained with adequate follow up. While the findings are compelling regarding the possible benefits of local-regional therapies to limit HCC recurrence and mortality following OLT, prospective studies using consistent treatment algorithms and therapeutic endpoints to validate these findings is warranted. Additionally, future prospective studies with 
perhaps centralized radiographic review would allow study of whether radiographic response could predict overall pathologic response and thus potentially improve stratification of patients for OLT and liver allocation. Thus clear radiographic predictors of pathologic response in prospective studies may allow for allocation of livers to patients with active, viable tumor burdens whereas patients who exhibit a complete radiographic response may not require additional treatments.

\section{Acknowledgments}

The study was partly funded by a limited grant from Biocompatibles Inc., a BTG International group company and by R01-DK070869 (DES) from the National Institutes of Health (NIDDK). The authors are grateful to the many patients who were subjects for this study. We also wish to thank the many nurses, advanced care providers, and physicians who participated in the care of these patients.

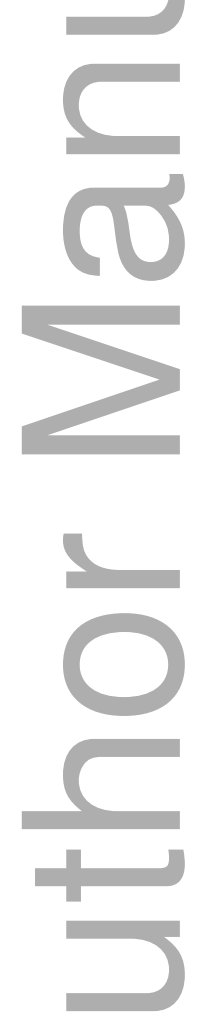

Figure Legends

Figure 1. Survival rate (event = death) by time (in months) following OLT for HCC.

Figure 2. Relationship between maximum tumor size on explant pathology and log hazard ratio (HR) for HCC recurrence. Results were obtained through a Cox regression model (containing all 
adjustment covariates) and five categories for tumor size (cm). Each log HR is plotted against its respective tumor size.

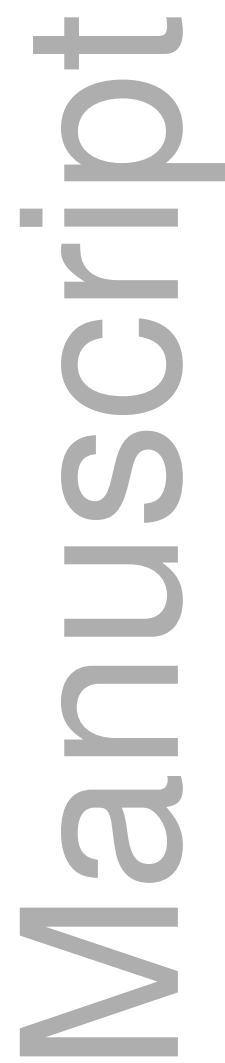

\section{References}

1. Sawyers CL, Abate-Shen C, Anderson KC, Barker A, Baselga J, Berger NA, et al. AACR Cancer Progress Report 2013. Clin Cancer Res. 2013;19(20 Suppl):S4-98.

2. Piscaglia F, Svegliati-Baroni G, Barchetti A, Pecorelli A, Marinelli S, Tiribelli C, et al. Clinical patterns of hepatocellular carcinoma in nonalcoholic fatty liver disease: A multicenter prospective study. Hepatology (Baltimore, Md. 2016;63(3):827-38.

3. El-Serag HB, Kanwal F, Richardson P, and Kramer J. Risk of hepatocellular carcinoma after sustained virological response in Veterans with hepatitis $C$ virus infection. Hepatology (Baltimore, Md. 2016;64(1):130-7.

4. El-Serag HB. Hepatocellular carcinoma. The New England journal of medicine. 2011;365(12):1118-27.

5. Wedd JP, Nordstrom E, Nydam T, Durham J, Zimmerman M, Johnson T, et al. Hepatocellular carcinoma in patients listed for liver transplantation: Current and future allocation 
policy and management strategies for the individual patient. Liver Transpl. 2015;21(12):154352.

6. Salvalaggio PR, Felga G, Axelrod DA, Della Guardia B, Almeida MD, and Rezende MB. List and liver transplant survival according to waiting time in patients with hepatocellular carcinoma. Am J Transplant. 2015;15(3):668-77.

7. Heimbach JK, Hirose R, Stock PG, Schladt DP, Xiong H, Liu J, et al. Delayed hepatocellular carcinoma model for end-stage liver disease exception score improves disparity in access to liver transplant in the United States. Hepatology (Baltimore, Md. 2015;61(5):164350.

8. Schlansky B, Chen Y, Scott DL, Austin D, and Naugler WE. Waiting time predicts survival after liver transplantation for hepatocellular carcinoma: a cohort study using the United Network for Organ Sharing registry. Liver Transpl. 2014;20(9):1045-56.

9. Terzi E, Ray Kim W, Sanchez W, Charlton MR, Schmeltzer P, Gores GJ, et al. Impact of multiple transarterial chemoembolization treatments on hepatocellular carcinoma for patients awaiting liver transplantation. Liver Transpl. 2015;21(2):248-57.

10. Tsochatzis E, Garcovich M, Marelli L, Papastergiou V, Fatourou E, RodriguezPeralvarez ML, et al. Transarterial embolization as neo-adjuvant therapy pretransplantation in patients with hepatocellular carcinoma. Liver Int. 2013;33(6):944-9.

11. Kulik L, Heimbach JK, Zaiem F, Almasri J, Prokop LJ, Wang Z, et al. Therapies for Patients with Hepatocellular Carcinoma Awaiting for Liver Transplantation: a Systematic Review and Meta-analysis. Hepatology (Baltimore, Md. 2017.

12. Salvalaggio PR, Felga GE, Alves JA, Meirelles RF, Jr., Almeida MD, and de Rezende MB. Response to transarterial chemoembolization in candidates with hepatocellular carcinoma within Milan criteria does not predict post-transplant disease-free survival. Transplantation proceedings. 2014;46(6):1799-802.

13. Kim WR, Smith JM, Skeans MA, Schladt DP, Schnitzler MA, Edwards EB, et al. OPTN/SRTR 2012 Annual Data Report: liver. Am J Transplant. 2014;14 Suppl 1:69-96.

14. Lewin SM, Mehta N, Kelley RK, Roberts JP, Yao FY, and Brandman D. Liver transplantation recipients with nonalcoholic steatohepatitis have lower risk hepatocellular carcinoma. Liver Transpl. 2017;23(8):1015-22.

15. Merani S, Majno P, Kneteman NM, Berney T, Morel P, Mentha G, et al. The impact of waiting list alpha-fetoprotein changes on the outcome of liver transplant for hepatocellular carcinoma. Journal of hepatology. 2011;55(4):814-9.

This article is protected by copyright. All rights reserved 
16. Agopian VG, Morshedi MM, McWilliams J, Harlander-Locke MP, Markovic D, Zarrinpar A, et al. Complete pathologic response to pretransplant locoregional therapy for hepatocellular carcinoma defines cancer cure after liver transplantation: analysis of 501 consecutively treated patients. Annals of surgery. 2015;262(3):536-45; discussion 43-5.

17. Llovet JM, Real MI, Montana X, Planas R, Coll S, Aponte J, et al. Arterial embolisation or chemoembolisation versus symptomatic treatment in patients with unresectable hepatocellular carcinoma: a randomised controlled trial. Lancet. 2002;359(9319):1734-9.

18. Bruix J, Reig M, and Sherman M. Evidence-Based Diagnosis, Staging, and Treatment of Patients With Hepatocellular Carcinoma. Gastroenterology. 2016;150(4):835-53.

19. Bruix J, Takayama T, Mazzaferro V, Chau GY, Yang J, Kudo M, et al. Adjuvant sorafenib for hepatocellular carcinoma after resection or ablation (STORM): a phase 3 , randomised, double-blind, placebo-controlled trial. Lancet Oncol. 2015;16(13):1344-54. 20. Huang J, Yan L, Cheng Z, Wu H, Du L, Wang J, et al. A randomized trial comparing radiofrequency ablation and surgical resection for $\mathrm{HCC}$ conforming to the Milan criteria. Annals of surgery. 2010;252(6):903-12.

21. Golfieri R, Cappelli A, Cucchetti A, Piscaglia F, Carpenzano M, Peri E, et al. Efficacy of selective transarterial chemoembolization in inducing tumor necrosis in small $(<5 \mathrm{~cm})$ hepatocellular carcinomas. Hepatology (Baltimore, Md. 2011;53(5):1580-9.

22. Prentice RL, and Kalbfleisch JD. Mixed discrete and continuous Cox regression model. Lifetime Data Anal. 2003;9(2):195-210.

23. Shetty K, Timmins K, Brensinger C, Furth EE, Rattan S, Sun W, et al. Liver transplantation for hepatocellular carcinoma validation of present selection criteria in predicting outcome. Liver Transpl. 2004;10(7):911-8.

24. Hameed B, Mehta N, Sapisochin G, Roberts JP, and Yao FY. Alpha-fetoprotein level > $1000 \mathrm{ng} / \mathrm{mL}$ as an exclusion criterion for liver transplantation in patients with hepatocellular carcinoma meeting the Milan criteria. Liver Transpl. 2014;20(8):945-51.

25. Harper AM, Edwards E, Washburn WK, and Heimbach J. An early look at the Organ Procurement and Transplantation Network explant pathology form data. Liver Transpl. 2016;22(6):757-64.

26. Vibert E, Azoulay D, Hoti E, lacopinelli S, Samuel D, Salloum C, et al. Progression of alphafetoprotein before liver transplantation for hepatocellular carcinoma in cirrhotic patients: a critical factor. Am J Transplant. 2010;10(1):129-37.

27. Vauthey JN, Lauwers GY, Esnaola NF, Do KA, Belghiti J, Mirza N, et al. Simplified staging for hepatocellular carcinoma. J Clin Oncol. 2002;20(6):1527-36. 
28. Fan L, Mac MT, Frishberg DP, Fan X, Dhall D, Balzer BL, et al. Interobserver and intraobserver variability in evaluating vascular invasion in hepatocellular carcinoma. $J$ Gastroenterol Hepatol. 2010;25(9):1556-61.
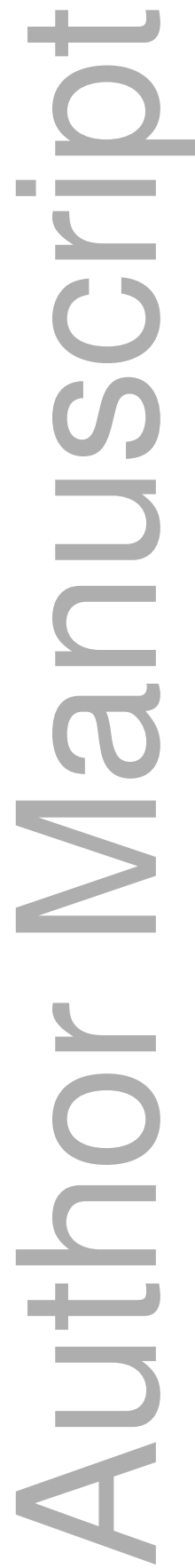

This article is protected by copyright. All rights reserved 
Table 1. Descriptive statistics on study cohort ( $n=441$ patients).

\begin{tabular}{|l|l|}
\hline Characteristic & Median $\mathbf{5}^{\text {th }}$ and $\mathbf{9 5}^{\text {th }}$ percentile) or Percent \\
\hline Age (years) & $56(44,68)$ \\
\hline Female & $20.9 \%$ \\
\hline AFP initial presentation $(\mathrm{ng} / \mathrm{mL})$ & $15.5(2,569)$ \\
\hline AFP at OLT $(\mathrm{ng} / \mathrm{mL})$ & $19(2,437)$ \\
\hline Lab MELD at OLT & $13(7,27)$ \\
\hline MELD Difference $(\mathrm{HCC}$ exception $-\mathrm{Lab})$ & $9.4(0,20)$ \\
\hline Tumor size: initial presentation & $2.5 \mathrm{~cm} \mathrm{(1,4.9)}$ \\
\hline Tumor number: initial presentation & $1(0.8)$ \\
\hline Tumor size: explant pathology & $2.0 \mathrm{~cm}(0,5)$ \\
\hline Tumor number: explant pathology & $1(1.7)$ \\
\hline Time from initial presentation to OLT & $6.5 \mathrm{months}(1.4,39.1)$ \\
\hline Etiology & \\
\hline Cryptogenic & $5.0 \%$ \\
\hline ETOH & $8.8 \%$ \\
\hline HBV & $7.7 \%$ \\
\hline HCV & $69.0 \%$ \\
\hline NASH & $5.4 \%$ \\
\hline PSC/PBC/AlH & $2.3 \%$ \\
\hline Other & $1.3 \%$ \\
\hline Local-regional treatment, $\mathrm{n}(\%)$ & $238(54 \%)$ \\
\hline TACE & $170(71 \%)$ \\
\hline RFA & $51(21 \%)$ \\
\hline TACE and RFA & $17(7 \%)$ \\
\hline Death & $30.0 \%$ \\
\hline HCC Recurrence & $7.9 \%$ \\
\hline & \\
\hline
\end{tabular}

This article is protected by copyright. All rights reserved 
Table 2. Cox regression analysis for HCC recurrence rate following OLT.

Values presented for each model are hazard ratios $(\mathrm{HR})$, with bold indicating significant $(p<0.05)$ and underlined indicating "near-significant" $(0.05<p<0.10)$.

\begin{tabular}{|l|c|c|c|c|c|}
\hline Characteristic & Model 1 & Model 2 & Model 3 & Model 4 & Model 5 \\
\hline Age (per 5 years) & 0.97 & 1.01 & 1.00 & 0.97 & 0.98 \\
\hline AFP at OLT (per 1,000 ng/mL) & 1.00 & 1.00 & 1.00 & 1.00 & 1.00 \\
\hline AFP: rate of increase & -- & 0.99 & 0.99 & 0.99 & -- \\
\hline Sex = female & 1.34 & 2.12 & 1.80 & 1.24 & 1.33 \\
\hline MELD at OLT & 0.98 & 1.01 & 0.98 & 1.00 & 0.97 \\
\hline $\begin{array}{l}\text { MELD Difference } \\
\text { (exception - lab) }\end{array}$ & $\mathbf{0 . 9 3}$ & 1.01 & 0.95 & 0.95 & 0.95 \\
\hline Tumor size: initial presentation & $\mathbf{1 . 3 6}$ & -- & $\mathbf{1 . 5 3}$ & -- & $\mathbf{1 . 4 5}$ \\
\hline $\begin{array}{l}\text { Tumor number: initial } \\
\text { presentation }\end{array}$ & 0.99 & -- & 1.06 & -- & 1.08 \\
\hline Tumor size; explant pathology & -- & $\mathbf{1 . 7 3}$ & -- & --- & -- \\
\hline $\begin{array}{l}\text { Tumor number: explant } \\
\text { pathology }\end{array}$ & -- & 1.08 & -- & -- & -- \\
\hline $\begin{array}{l}\text { Progression (tumor size } \\
\text { increase) }\end{array}$ & -- & -- & $\mathbf{6 . 3 2}$ & -- & -- \\
\hline $\begin{array}{l}\text { Progression (tumor number } \\
\text { increase) }\end{array}$ & -- & -- & 1.11 & -- & -- \\
\hline $\begin{array}{l}\text { Time from initial presentation } \\
\text { to OLT }\end{array}$ & 1.01 & 1.00 & 1.00 & 1.00 & 1.01 \\
\hline Treatment (vs. no treatment) & -- & $\mathbf{0 . 8 0}$ & 0.87 & $\mathbf{0 . 8 1}$ & -- \\
\hline \begin{tabular}{l} 
RFA \\
\hline TACE
\end{tabular} & -- & -- & -- & -- & 0.85 \\
\hline $\begin{array}{l}\text { RFA and TACE } \\
\text { (palendar year of diagnosis }\end{array}$ & -- & $\mathbf{4 . 5 4}$ & -- & -- & -- \\
\hline Microvascular invasion & -- & -- & $\mathbf{0 . 3 6}$ \\
\hline
\end{tabular}

This article is protected by copyright. All rights reserved 
Table 3. Cox regression analysis for mortality rate following OLT.

Values presented for each model are hazard ratios $(\mathrm{HR})$, with bold indicating significant $(p<0.05)$ and underlined indicating "near-significant" $(0.05<p<0.10)$

\begin{tabular}{|l|c|c|c|}
\hline Characteristic & Model 1 & Model 2 & Model 3 \\
\hline Age (per 5 yrs) & $\mathbf{1 . 1 6}$ & $\mathbf{1 . 1 6}$ & $\mathbf{1 . 1 9}$ \\
\hline AFP at OLT (per 1,000 ng/mL) & $\mathbf{1 . 1 2}$ & 1.00 & 1.11 \\
\hline AFP: rate of increase & -- & $\underline{3.42}$ & 1.19 \\
\hline Sex = female & 1.45 & $\mathbf{1 . 5 9}$ & 1.51 \\
\hline MELD at OLT & $\underline{1.03}$ & 1.03 & 1.02 \\
\hline $\begin{array}{l}\text { Difference in MELD } \\
\text { (exception - lab) }\end{array}$ & 0.97 & $\underline{0.99}$ & $\underline{0.96}$ \\
\hline Tumor size: initial presentation & 1.07 & -- & 1.06 \\
\hline Tumor number: initial presentation & $\mathbf{1 . 2 6}$ & -- & $\mathbf{1 . 2 8}$ \\
\hline Tumor size: explant pathology & -- & $\mathbf{1 . 1 9}$ & -- \\
\hline Tumor number: explant pathology & -- & 1.09 & $\mathbf{1 . 2 8}$ \\
\hline Progression (tumor size increase) & 1.47 & -- & 1.47 \\
\hline Progression (tumor number increase) & $\mathbf{1 . 4 1}$ & -- & $\mathbf{1 . 4 1}$ \\
\hline Time from initial presentation to OLT & 1.01 & 1.01 & 1.01 \\
\hline Treatment (vs. no treatment) & & & \\
\hline RFA & -- & -- & -- \\
\hline TACE & -- & -- & -- \\
\hline RFA and TACE & -- & -- & -- \\
\hline Calendar year of diagnosis (per year) & -- & 1.01 & 1.05 \\
\hline Microvascular invasion & -- & $\underline{1.51}$ & -- \\
\hline
\end{tabular}

This article is protected by copyright. All rights reserved 
Table 4. Linear regression analysis for predictors of tumor size at OLT explant pathology.

Values presented for each model represent estimated increase (greater than zero) or decrease (less than zero) in maximum tumor size $(\mathrm{cm})$ per unit increase in the covariate as indicated, covariate-adjusted. Note that bold indicates significant $(p<0.05$, with underlined indicating "nearsignificant" $(0.05<p<0.10)$.

\begin{tabular}{|l|c|c|}
\hline Characteristic & Model 1 & Model 2 \\
\hline Age (per 5 yrs) & 0.005 & 0.006 \\
\hline AFP at OLT (per 1,000 ng/mL) & 0.019 & 0.075 \\
\hline Sex = female & -0.16 & -0.13 \\
\hline MELD at OLT & $\underline{0.026}$ & 0.014 \\
\hline $\begin{array}{l}\text { Difference in MELD } \\
\text { (exception - lab) }\end{array}$ & -0.024 & -0.024 \\
\hline Tumor size: initial presentation & $\mathbf{0 . 2 7}$ & $\mathbf{0 . 3 1}$ \\
\hline Time from initial presentation to OLT & -0.004 & -0.000 \\
\hline Treatment (vs. none) & & \\
\hline RFA & -- & $-\mathbf{0 . 5 6}$ \\
\hline TACE & -- & $\mathbf{- 0 . 6 3}$ \\
\hline RFA and TACE & -- & $\mathbf{- 1 . 6 2}$ \\
\hline Calendar year of diagnosis (per year) & $\mathbf{- 0 . 1 3}$ & $\mathbf{- 0 . 0 8 4}$ \\
\hline
\end{tabular}

This article is protected by copyright. All rights reserved 


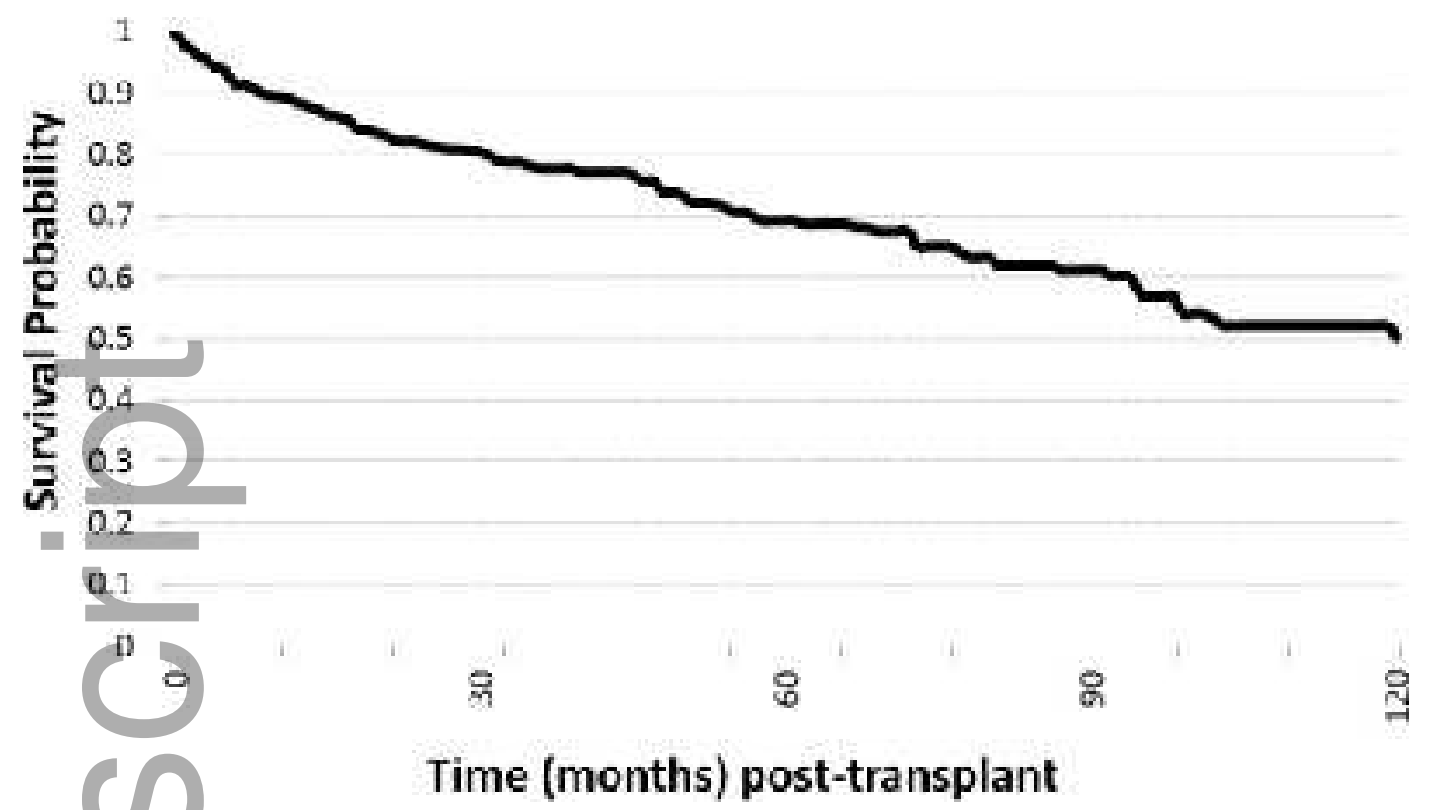

lt_25194_f1.tif

This article is protected by copyright. All rights reserved 


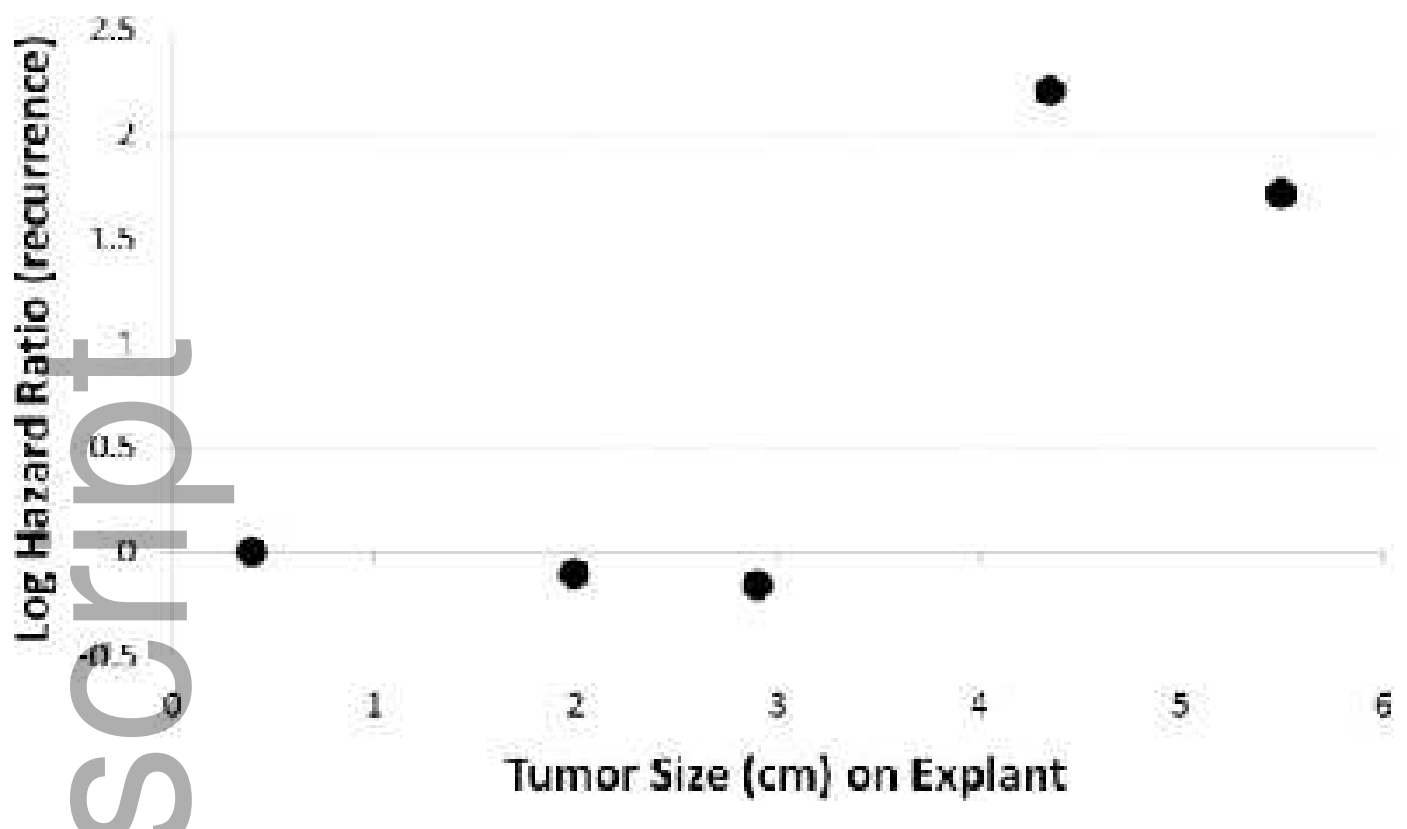

lt_25194_f2.tif

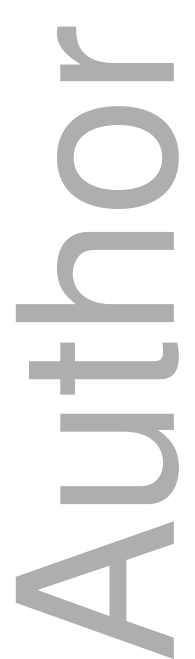

This article is protected by copyright. All rights reserved 